\title{
Logical appropriateness as a variable in a compound paired-associate learning task'
}

\author{
Robert E. Shaw and James H. Koplin \\ VANDERBILT UNIVERSITY
}

\begin{abstract}
In a compound response paired-associate learning task (e.g., blue- then color) 20 Ss made more correct anticipations when the connective coupling the response to the stimulus word was logically appropriate for the word-pair class to which the stimulus and response words belong than when it was logically inappropriate. The appropriateness value of each connective was computed as the relative frequency with which 45 Ss selected it as the best connective to use between each wordpair class.
\end{abstract}

\section{Problem}

Conventional linguistic labels for logical relationships have been agreed upon by logicians. They appear in the standard introductory textbooks on symbolic logic (see for example, Suppes, 1957, p. 3). The English language equivalents for the logical connectives which name the most fundamental relationships are "and," "or,", "if,", "then," and "not." Thus far no empirical ties have been discovered between the theoretical logical relationships and the learning and performance variables of human Ss. This experiment attempted to establish one such tie in a standard laboratory task.

One method of analyzing the normative data gathered in a free association situation has been to classify the stimuli and responses into word pairs which are defined by simple logical relationships. Some of the classes often used are coordinates (cheese-crackers), ordinate superordinate (blue-color), noun opposite (man-woman), and adjective opposite (hot-cold). Certain other classes of word pairs can be arbitrarily constructed by coupling members of different word form classes. This leads to such cases as adjective-noun (red-ball), noun-adjective (sky-blue), and noun-verb (river-flows). This does not exhaust the possible pairs that can be constructed; the seven classes above were chosen for this experiment because they represent the most obvious combinations. If a particular logical relationship is specific to each of the word-pair classes, then certain logical connectives should be more appropriate for one class of pairs than for another. From these considerations it was predicted that Ss would be able consistently to assign preferential rankings to the English equivalents of the connectives regarding their appropriateness for each of the seven classes. These rankings permit the calculation of a behaviorally defined "appropriateness" value for each connective for each class. Given this information, material could be constructed for a compound response paired-associate (PA) learning task to test the hypothesis that Ss would make more correct anticipations when the connective coupling the response to the stim- ulus word was logically appropriate for the wordpair class to which the stimulus and response words belong than when it is logically inappropriate. Support for this hypothesis would suggest the possibility of developing a logical model which would clarify the nature of the associative relationship between fundamental linguistic units in terms of the logical relationships formally defined in the propositional calculus.

\section{Method}

The experiment was conducted in two phases. In Phase I $45 \mathrm{Ss}$ were presented with a completion test composed of 110 stimulus-response word pairs from the Minnesota norms (Russell \& Jenkins, 1954) with a blank space between each pair. Ss were instructed to fill in the blank with the connective word that best suited each pair of words. A pool of logical connectives was presented at the top of each page: and, then, not, if, and or. A typical pair might be "doctor nurse," and a typical S might insert "or" in the blank. The "logical appropriateness" of each connective was then computed as the relative frequency (expressed as a percentage) of that connective as chosen by the $45 \mathrm{Ss}$ for each class of word pairs. This measure ranged from $78 \%$ for the connective "and" in the coordinates class to $.2 \%$ for "if" in the noun-opposite class of word pairs.

The normative data were used in Phase II. Twenty Ss learned a 32-item PA list composed of eight items in each of four categories. Some sample items are presented in Table 1. Notice that the stimulus is one word, while the response is composed of a connective and a word. These response units were constructed so that half the words had a high associative relationship to the stimulus and half a low associative relationship. The mean association strength for the 16 high units was 552.3 and for the low units it was 63.3. Half of the pairs contained a highly appropriate connective and half a low appropriateness connective. As described above appropriateness can vary on a scale from 0 to 100 . The

Table 1. Some Sample Pairs of the Types Used in the PairedAssociate Learning List

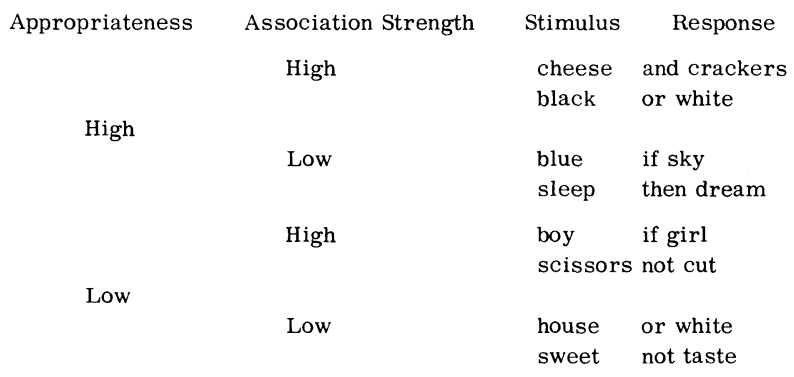




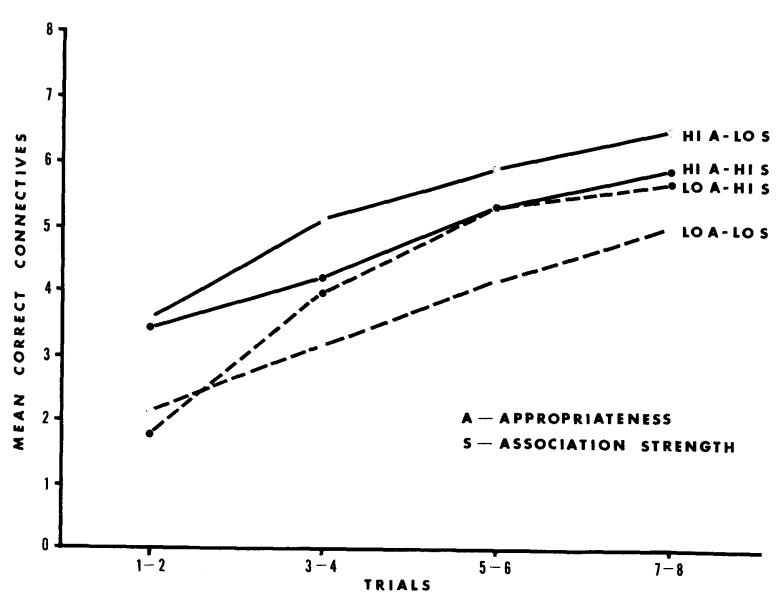

Fig. 1. Mean correct anticipations of the logical connectives.

average for the high pairs was 43.2 and for the low pairs 4.2. Since appropriateness is a function of the wordform class membership of the stimulus and response words, as much ambiguity as possible was avoided by choosing only stimulus and response words which were strong members of a particular form-class.

Three randomizations of the pairs were presented for one practice and eight anticipation trials, using a Lafayette memory drum. Standard paired-associate learning instructions were modified for compound responses.

\section{Hesults and Discussion}

Each $\mathrm{S}$ was scored for the number of correct connectives and for the number of correct response words by type of pair on each of the eight anticipation trials. The mean performance on the connectives for all $20 \mathrm{Ss}$ is plotted in two-trial blocks in Fig. 1.

Inspection of the figure indicates that appropriateness is a significant effect. This is substantiated by the results of an A (4 types of pairs) X B (trials) X S (20 Ss) analysis of variance. Type of Pair is a significant main effect ( $F, 20.21$; df, 3/57; $\mathrm{p}<.001$ ). The trials effect is significant ( F, 79.94; df, 7/133; $p<.001$ ); and the interaction of trials by type of pair is significant (F, 3.49; df, 21/399; $\mathrm{p}<.001$ ). The latter indicates that the four types of pairs were learned at different rates.

Multiple comparisons among the four types of pairs showed that high and low appropriateness connectives were significantly different (F,47.80; df, 1/57; $<<.001)$. The combined high association strength pairs did not differ from low association strength pairs $(F<1$; df, 1/57). The interaction of association strength and logical appropriateness is significant ( F, 12.79; df, 1/57; $p<.001)$. Examination of the curves in Fig. 1 shows that the interaction arises from the ease of learning of the low association strength and high appropriateness type of pair. Logical appropriateness has its strongest effect, then, when associative strength is low. The usual hypothesis would be that the effects of the two variables would combine to yield marked facilitation. However, it is the High A-Low S pairs which are most easily learned. A replication of the experiment has confirmed this. There is no obvious explanation. The lower scores for the High A-Low S pairs could possibly be due to complex interference effects among the several types of pairs in the PA list. Further examination of this interaction is planned in an experiment in which one group learns only High A pairs and another only Low A pairs.

A similar analysis of the response word data roughly approximates the above pattern of results. The only difference is that the multiple comparisons show association strength to be significant. This must be interpreted in conjunction with the significant interaction; an inspection of the data shows that the High A-Low $S$ type of pair is still superior, but relatively reduced, but the Low A-Low $\mathrm{S}$ type of pair is markedly inferior.

The results confirm the hypothesis that logical appropriateness is a major factor in this compound response paired-associate learning task. This is the most important point to be made in interpreting the experiment.

Though this experiment considered only the logical relationships between pairs of words, a logical model of language derived from the propositional calculus should be able to handle the relationships among larger units of language such as phrases or sentences. Such a model seems plausible since the propositional calculus (Hilbert \& Ackermann, 1950) provides a method for analyzing linguistic units regardless of complexity into a sequence of simple single-valued terms joined by the fundamental logical relationships. The learning of such a sequence of constituent terms should be facilitated or impeded according to the degree of appropriateness of the connectives coupling the terms. This empirical question can best be attacked by attempting to construct such a logical model of the flow of discourse which will suggest testable hypotheses.

\section{Referenees}

HILBERT, D., \& ACKERMANN, W. Principles of mathematical logic. New York: Chelsea, 1950. RUSSELL, W. A., \& JENKINS, J. J. The complete Minnesota norms for responses to 100 words from the Kent-Rosanoff word association test. Tech. Rep. No. 11, SN onr 66216, Univer. of Minnesota, 1954. SUPPES, P. Introduction to logic. Princeton: D. Van Nostrand, 1957.

\section{Note}

1. This work was supported, in part, by an NIMH predoctoral fellowship ( $\mathrm{MH}-24,010)$ awarded to the first author sponsored by the second author. A paper based on this experiment was presented at the SEPA convention, Gatlinburg, Tennessee, April, 1964. A copy of the SEPA paper which extends the scope of the proposed logical model is available upon request. 